\title{
Pulsed Eddy-Current Response to a Conducting Half-Space
}

\author{
John Bowler, Member, IEEE, and Marcus Johnson
}

\begin{abstract}
Eddy-current nondestructive evaluation is commonly carried out using single frequency time harmonic excitations, but a pulsed excitation offers a simple and effective alternative. The pulse signals have been calculated for a probe coil whose current rises and falls exponentially, approximating a square wave when the exponential time constant is small. Predictions of the induced electromotive force (EMF) across a coil above a half-space conductor and of the magnetic field on the coil axis have been compared with experiments. The comparison shows excellent agreement between theory and experiment.
\end{abstract}

Index Terms - Eddy current, half-space, pulsed, transient.

\section{INTRODUCTION}

$\mathbf{P}$ ULSED eddy-current measurements rival single and multifrequency testing for many of the common applications in nondestructive evaluation such as the detection of defects, the measurement of conductivity, and the estimation of the thickness of coatings. The basic advantages of a transient system are, first, that the circuitry is relatively simple compared with that needed for broad band alternating current testing and, second, that a single transient response contains as much information as an entire spectrum of frequency domain excitations. In order to extract the information and thus realize the full potential of pulsed eddy-current testing, the signals must first be analyzed. As an aid to transient signal analysis, this paper examines some basic aspects of the theory of pulsed eddy-current testing.

Theoretically, a transient field or an induced voltage is related to the corresponding time-harmonic complex amplitude through the Laplace transform. Consequently, results from an analysis of alternating fields can be used to determine the variation of the probe signal or the electromagnetic field with time. In order to evaluate the time domain field or signal it is necessary to carry out an inverse Laplace transform, but an exact inverse is not always available. For example, the frequency dependence may be too complicated or simply not of a form that can be integrated by exact methods. One possible recourse is to express the original solution in terms of a Fourier transform rather than a Laplace transform and compute the time dependence numerically using a fast Fourier transform (FFT) [1]. However, in the present study, the inverse

Manuscript received May 6, 1996; revised October 18, 1996. This work was supported by the Ministry of Defence and the Structural Materials Centre, DRA, Farnborough, U.K.

The authors are with the Department of Physics, University of Surrey, Guildford, Surrey, U.K.

Publisher Item Identifier S 0018-9464(97)02089-X.
Laplace transforms are reducible to standard forms and can be evaluated exactly using contour integration.

Our starting point is the frequency domain electromagnetic field due to a circular current filament whose axis is normal to the surface of a uniform half-space conductor. This field, given by Wait [2] and later by Hammond [3], is expressed in the form of spatial frequency integrals of the Sommerfeld type involving the first-order Bessel function. A superposition of the filament solutions gives the time-harmonic field due to a coil of rectangular cross section [4]. For a half-space conductor, the resulting equation can be transformed into the time domain analytically. Here the Sommerfeld spatial frequency integration is carried out using a standard numerical method [5] to give the transient coil electromotive force (EMF) and the electromagnetic field.

Closely related problems to those that arise in eddy-current testing occur in the theory of geophysical surveying where the interaction of electromagnetic fields with the earth is studied. In this context, Wait has derived an expression for the transient field due to a filamentary coil excited by an instantaneous step current [6]. Following Wait, a number of others have considered the same solution [7], [8]. In the present paper, a more general problem is considered in which transient signals for coils of finite cross section have been calculated by considering a pulse excitation with a finite rise time. This is a simple generalization of the idealized case of a unit step current excitation [9].

\section{COIL EMF}

Consider a coil of rectangular cross section whose axis is normal to the surface of the conductor. The coil carries a prescribed current varying in time as

$$
I(t)=I_{0}\left(1-e^{-t / \tau_{0}}\right) u(t)
$$

where $\tau_{0}$ is the characteristic rise time of the current, and $u(t)$ is a unit step function. $u(t)=1$ for $t>0$ but is otherwise zero. The induced current in the conductor, generated by the changing flux surrounding the coil, increases initially but then decays to zero as its energy is dissipated and the primary magnetic field due to the coil tends toward a steady state. By sampling the coil EMF as a function of time, the relaxation of the eddy-current field can be measured and the observations related to the probe and material parameters. For example, it is possible to infer the conductivity of the material from the probe signal. In making precise measurements, practical 


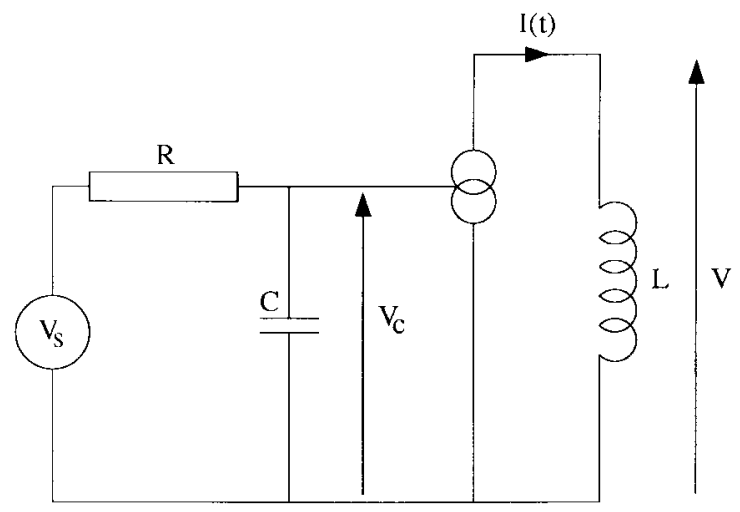

Fig. 1. Equivalent excitation coil circuit.

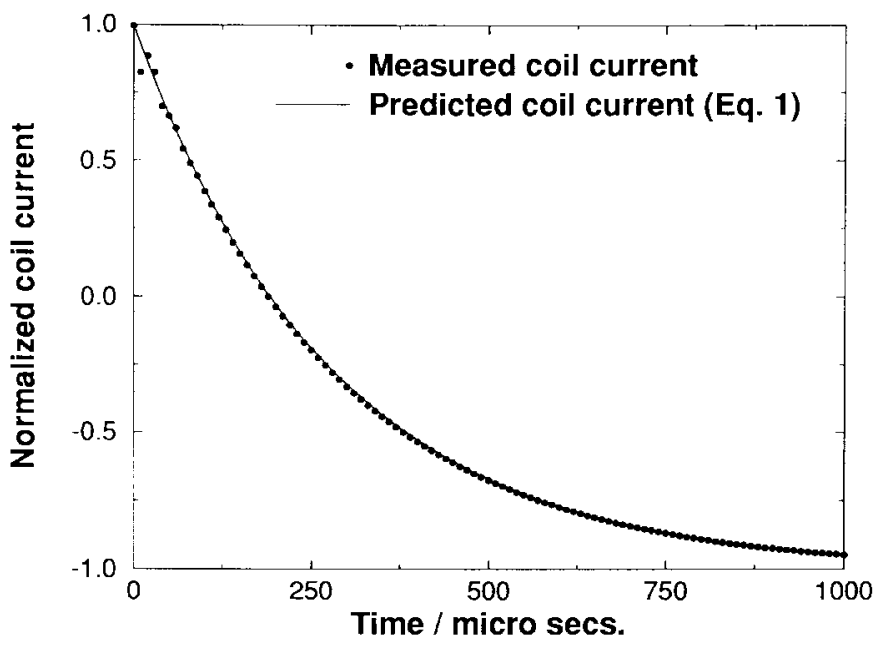

Fig. 2. Measured coil current normalized between \pm 1 and compared with that given by (1) for $\tau_{0}=275 \mu \mathrm{s}$.

problems arise due to ringing of the coil EMF. Ringing occurs at the probe's resonant frequency which is lowered due to stray capacitance in the leads. The effect can be mitigated by increasing the excitation time constant $\tau_{0}$.

An idealized equivalent circuit of the system, shown in Fig. 1 , is applicable to the case where the probe is in free space. $V_{\mathrm{S}}$ is a bipolar square wave, the amplitude of which determines the amplitude of the coil current $I(t)$. The time variation of the coil current $\tau_{0}$ in (1) is determined by a first-order RC filter, the values of $\mathrm{R}$ and $\mathrm{C}$ being $4048 \Omega$ and $0.068 \mu \mathrm{F}$, respectively. A transconductance amplifier ensures that the coil current is proportional to the filter output voltage and is independent of the series resistance of the coil. Fig. 2 shows a comparison of the measured coil current and that predicted using (1) for a time constant $\tau_{0}$ of $275 \mu \mathrm{s}$ where $\tau_{0}$ is equal to the product of $\mathrm{R}$ and $\mathrm{C}$ in Fig. 1.

If the coil resistance is neglected, then the coil current and the induced EMF across the coil will vary as shown in Fig. 3. Also shown in Fig. 3 is the coil EMF as a function of time in the presence of a conducting workpiece. The difference between the two voltage transients, in free space and on the conductor, is due to the induced EMF arising from the eddycurrent field as shown in Fig. 4. Circuit theory alone is not

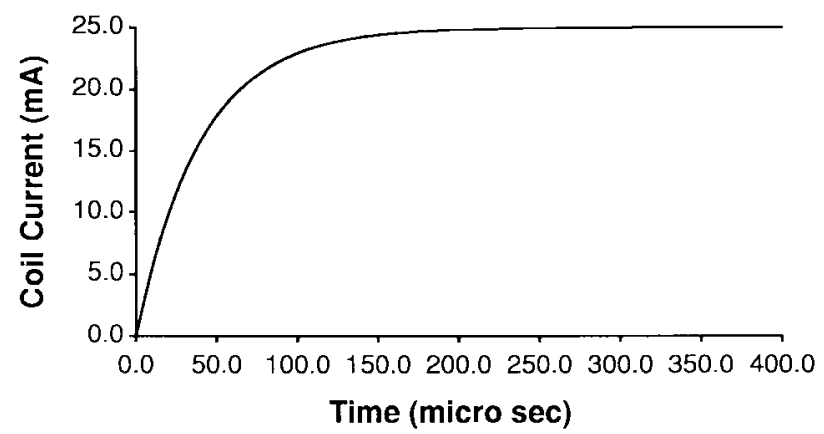

(a)

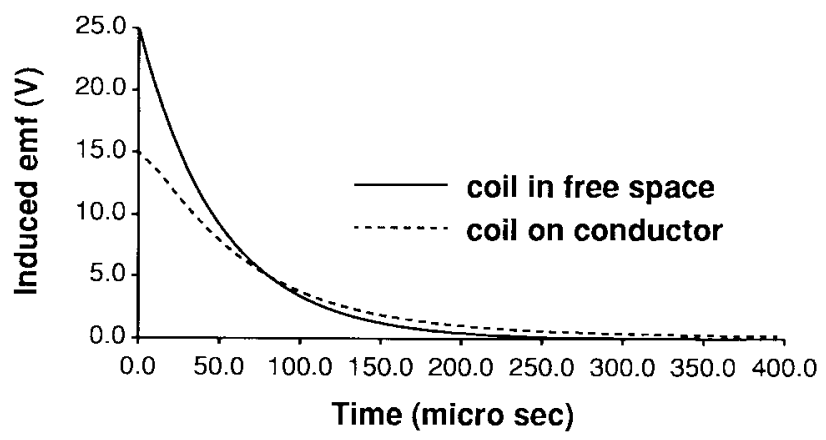

(b)

Fig. 3. (a) Coil current as a function of time with a source time constant $\tau_{0}=20 \mu \mathrm{s}$. (b) Coil EMF variation as a function of time in free space, solid line, and in the presence of a conductor dashed line.

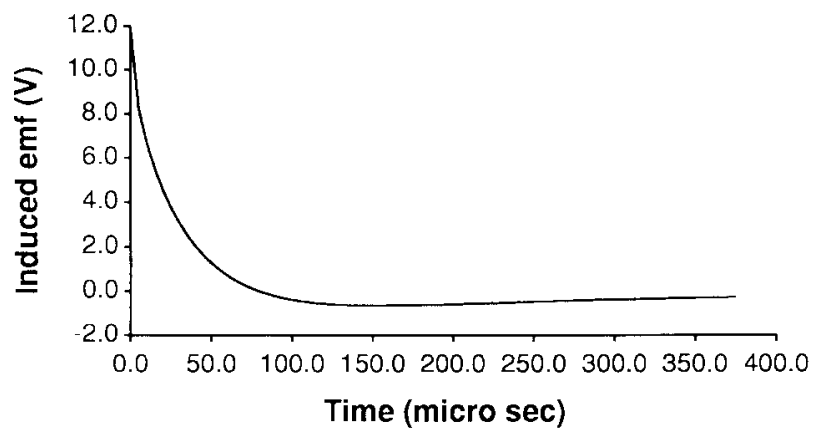

(a)

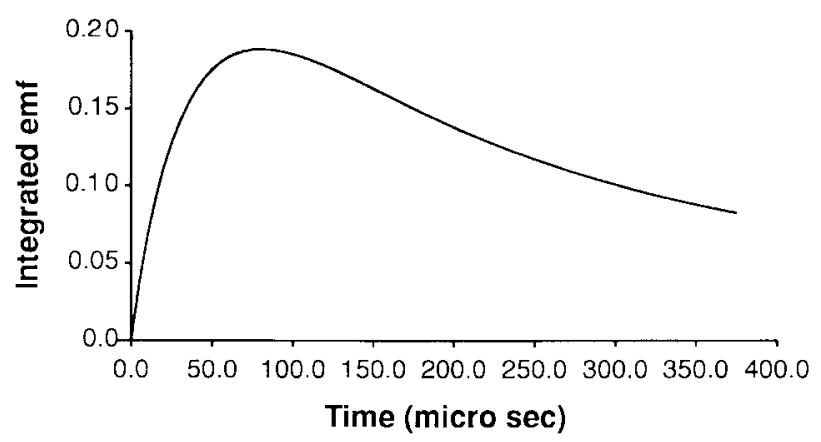

(b)

Fig. 4. (a) Eddy-current signal defined as the induced EMF for the coil in free space minus the induced EMF in the presence of the conductor. (b) Integrated eddy-current response.

suited to the prediction of the coil EMF due to induced currents or the response of a Hall sensor located on the coil axis. Instead the problem must be examined using field theory. 


\section{FIELD THEORY}

The required solutions of Maxwell's equations may be found by using the Laplace transform, written as

$$
f(s)=\int_{0}^{\infty} F(t) e^{-s t} d t .
$$

The corresponding inversion formula

$$
F(t)=\frac{1}{2 \pi i} \int_{B r} f(s) e^{s t} d s
$$

recovers the variation in time of the function $F(t)$ from its Laplace transform $f(s)$. The Bromwich contour $B r$ is the usual path of integration in the complex $s$ plane parallel to the imaginary axis, passing to the right of any poles. In the analysis below, the initial solutions considered are of the form $f(s) e^{s t}$. Equation (3) then synthesizes the time dependence from the corresponding $s$ domain solution. More commonly, the time dependence is defined by the real part of the phase factor $e^{-i \omega t}$, but the Laplace formalism means an $s$ appears in place of the $-i \omega$.

As is usual in eddy-current problems, the displacement current has been neglected. A coil carrying a current $\mathcal{I}(s)$, whose axis is normal to the surface of a half-space conductor and whose turns density is $N(\rho, z)$, excites an azimuthal electric field satisfying

$$
\begin{gathered}
{\left[\frac{\partial^{2}}{\partial \rho^{2}}+\frac{1}{\rho} \frac{\partial}{\partial \rho}-\frac{1}{\rho^{2}}+\frac{\partial^{2}}{\partial z^{2}}\right] E(s, \rho, z)=-\mu_{0} s \mathcal{I}(s) N(\rho, z)} \\
z>0
\end{gathered}
$$

in air and

$$
\left[\frac{\partial^{2}}{\partial \rho^{2}}+\frac{1}{\rho} \frac{\partial}{\partial \rho}-\frac{1}{\rho^{2}}+\frac{\partial^{2}}{\partial z^{2}}-s \mu \sigma\right] E(s, \rho, z)=0 \quad z<0
$$

in the conductor. It is not necessary to derive the complete solution because the time harmonic filament solution discussed by Hammond [3], and the more general case of a rectangular coil developed by Dodd and Deeds [4] forms the basic elements for all the results developed here. The elemental solution needed is the azimuthal electric field due to a circular current filament of radius $\rho^{\prime}$ at a height $z^{\prime}$ in air above a half-space conductor. The required field satisfies

$$
\begin{aligned}
{\left[\frac{\partial^{2}}{\partial \rho^{2}}+\frac{1}{\rho} \frac{\partial}{\partial \rho}-\frac{1}{\rho^{2}}+\frac{\partial^{2}}{\partial z^{2}}\right] } & \\
\cdot \stackrel{\circ}{G}\left(s, \rho, z \mid \rho^{\prime}, z^{\prime}\right) & =-\delta\left(\rho-\rho^{\prime}\right) \delta\left(z-z^{\prime}\right) / \rho \\
z & >0
\end{aligned}
$$

in air and

$$
\left[\frac{\partial^{2}}{\partial \rho^{2}}+\frac{1}{\rho} \frac{\partial}{\partial \rho}-\frac{1}{\rho^{2}}+\frac{\partial^{2}}{\partial z^{2}}-s \mu \sigma\right] \stackrel{\circ}{G}\left(s, \rho, z \mid \rho^{\prime}, z^{\prime}\right)=0
$$

in the conductor. From Hammond [3], the solution vanishing as $\rho \rightarrow \infty$ and $|z| \rightarrow \infty$ is

$$
\stackrel{\circ}{G}\left(s, \rho, z \mid \rho^{\prime}, z^{\prime}\right)=\int_{0}^{\infty} \widetilde{G}\left(\kappa, s, z, z^{\prime}\right) J_{1}\left(\kappa \rho^{\prime}\right) J_{1}(\kappa \rho) \kappa d \kappa
$$

where $J_{1}$ is a first order Bessel function of the first kind and

$\widetilde{G}\left(\kappa, s, z, z^{\prime}\right)= \begin{cases}\frac{1}{2 \kappa}\left[e^{-\kappa\left|z-z^{\prime}\right|}+\Gamma(\kappa, s) e^{-\kappa\left(z+z^{\prime}\right)}\right], & z>0 \\ \frac{1}{2 \kappa} T(\kappa, s) e^{\gamma\left(z+z^{\prime}\right)}, & z<0\end{cases}$

with $\gamma=\left(\kappa^{2}+s \mu \sigma\right)^{1 / 2}$, taking the root with a positive real part.

The field due to a filament has the properties of a Green's function in that its first-order derivative with respect to $z$ has a jump at $z=z^{\prime}$, and similarly the derivative with respect to $\rho$ is discontinuous at $\rho=\rho^{\prime}$. Consequently, the secondorder derivatives are symbolically related to delta functions. The half-space transverse electric reflection and transmission coefficients $\Gamma(\kappa, s)$ and $T(\kappa, s)$ are given by

$$
\Gamma(\kappa, s)=\frac{\mu_{r} \kappa-\gamma}{\mu_{r} \kappa+\gamma} \quad \text { and } \quad T(\kappa, s)=\frac{2 \mu_{r} \kappa}{\mu_{r} \kappa+\gamma} .
$$

Through the presence of these coefficients, the surface acts as a spatial frequency filter. At low spatial frequencies, the reflection coefficient approaches -1 and in the high spatial frequency limit, it tends to $\left(\mu_{r}-1\right) /\left(\mu_{r}+1\right)$. The transmission coefficient, on the other hand, increases from zero to $\mu_{r} /\left[\mu_{r}+\right.$ $1]$ as the spatial frequency increases from zero.

\section{COIL FIELD}

The field is linearly dependent on its source. Therefore, the superposition principle may be used to write the solution of (4) and (5) for the azimuthal electric field in terms of the solution of (6) and (7) as

$$
\begin{aligned}
E(s, \rho, z)= & \mu_{0} s \mathcal{I}(s) \int_{0}^{\infty} \int_{0}^{\infty} \stackrel{\circ}{G}\left(s, \rho, z \mid \rho^{\prime}, z^{\prime}\right) \\
& \cdot N\left(\rho^{\prime}, z^{\prime}\right) \rho^{\prime} d \rho^{\prime} d z^{\prime} .
\end{aligned}
$$

The electric field above the conductor may be written as

$$
E(s, \rho, z)=E_{0}(s, \rho, z)+\Delta E(s, \rho, z)
$$

separating the free space, $E_{0}(s, \rho, z)$, and reflection terms, $\Delta E(s, \rho, z)$. Combining (8), (9), (11), and (12) gives

$$
\begin{aligned}
E_{0}(s, \rho, z)= & \frac{1}{2} \mu_{0} s \mathcal{I}(s) \int_{0}^{\infty} \int_{0}^{\infty} \int_{0}^{\infty} e^{-\kappa\left|z-z^{\prime}\right|} \\
& \cdot N\left(\rho^{\prime}, z^{\prime}\right) J_{1}(\kappa \rho) J_{1}\left(\kappa \rho^{\prime}\right) d \kappa \rho^{\prime} d \rho^{\prime} d z^{\prime}
\end{aligned}
$$

and

$$
\begin{aligned}
\Delta E(s, \rho, z)= & \frac{1}{2} \mu_{0} s \mathcal{I}(s) \int_{0}^{\infty} \int_{0}^{\infty} \int_{0}^{\infty} \Gamma(\kappa, s) e^{-\kappa\left(z+z^{\prime}\right)} \\
& \cdot N\left(\rho^{\prime}, z^{\prime}\right) J_{1}(\kappa \rho) J_{1}\left(\kappa \rho^{\prime}\right) d \kappa \rho^{\prime} d \rho^{\prime} d z^{\prime} .
\end{aligned}
$$

Note that the integral over $\kappa$ in (13) can be evaluated in terms of elliptical integrals [10], using a standard form [11]. By changing the order of integration, (14) can be written

$$
\Delta E(s, \rho, z)=\frac{1}{2} \mu_{0} s \mathcal{I}(s) \int_{0}^{\infty} \Gamma(\kappa, s) e^{-\kappa z} \mathcal{J}(\kappa) J_{1}(\kappa \rho) d \kappa
$$

where $\mathcal{J}(\kappa)$ is defined in terms of integrals over the coil turns density function as

$$
\mathcal{J}(\kappa)=\int_{0}^{\infty} \int_{0}^{\infty} N(\rho, z) e^{-\kappa z} J_{1}(\kappa \rho) \rho d \rho d z
$$


which is a combination of a Hankel transform in the radial coordinate and a Laplace transform with respect to the $\mathrm{z}$ coordinate.

For a cylindrical coil of rectangular cross section and with the coil turns density $n$ constant over the cross section, we have

$$
N(\rho, z)= \begin{cases}n, & a_{2}<\rho<a_{1} \quad-b<z-h<b \\ 0, & \text { otherwise }\end{cases}
$$

Here $a_{1}$ is the outer radius of the coil and $a_{2}$ the inner radius. The axial length is $2 b$, and $h$ is the height of the coil center above the surface of the conductor. From (16) and (17) it is found that

$$
\mathcal{J}(\kappa)=\frac{2 n}{\kappa} e^{-\kappa h} \sinh (b \kappa)\left[a_{1}^{2} \chi\left(a_{1} \kappa\right)-a_{2}^{2} \chi\left(a_{2} \kappa\right)\right]
$$

where $\chi$, which arises from the radial integral of (16), is defined in terms of a standard form by [12]

$$
\begin{aligned}
\chi(\alpha) & =\int_{0}^{1} J_{1}(\alpha \rho) \rho d \rho \\
& =\frac{2 \pi}{\alpha}\left[J_{1}(\alpha) \mathbf{H}_{0}(\alpha)-J_{0}(\alpha) \mathbf{H}_{1}(\alpha)\right]
\end{aligned}
$$

where $\mathbf{H}_{0}$ and $\mathbf{H}_{1}$ are Struve functions.

\section{Time Domain FieldS}

It is evident from (15) that the frequency/time dependence of an arbitrary coil is determined by the product of the current and the reflection coefficient. Introducing the function

$$
\phi(\kappa, s)=\mathcal{I}(s) \Gamma(\kappa, s)
$$

the corresponding time variation and its time derivative are given by the inverse Laplace transforms

$$
\begin{aligned}
\Phi(\kappa, t) & =\frac{1}{2 \pi i} \int_{B r} \phi(\kappa, s) e^{s t} d s \\
\Phi^{\prime}(\kappa, t) & =\frac{1}{2 \pi i} \int_{B r} s \phi(\kappa, s) e^{s t} d s .
\end{aligned}
$$

For a half-space reflection coefficient and an exponential current variation with time, the integrals reduce to standard forms that can be evaluated analytically in terms of error functions.

Formally taking the inverse Laplace transform of (15), the time dependent reflected electric field is given by

$$
\Delta E(\rho, z, t)=\frac{1}{2} \mu_{0} \int_{0}^{\infty} \Phi^{\prime}(\kappa, t) e^{-\kappa z} \mathcal{J}(\kappa) J_{1}(\kappa \rho) d \kappa .
$$

The magnetic field is found from the induction law by taking the inverse Laplace transform of $-\nabla \cdot[\hat{\phi} \Delta E(s, \rho, z)] / \mu_{0} s, \hat{\phi}$ being the azimuthal unit vector. This gives

$$
\left[\begin{array}{l}
\Delta H_{\rho}(\rho, z, t) \\
\Delta H_{z}(\rho, z, t)
\end{array}\right]=-\frac{1}{2} \int_{0}^{\infty} \Phi(\kappa, t) e^{-\kappa z} \mathcal{J}(\kappa)\left[\begin{array}{l}
J_{1}(\kappa \rho) \\
J_{0}(\kappa \rho)
\end{array}\right] \kappa d \kappa
$$

for the reflected magnetic field above a uniform half-space conductor $(z>0)$. Equation (23) is evaluated using standard numerical techniques [5].

\section{PRobe Response}

The induced EMF in a probe coil in free space and the EMF due to the field reflected by the conductor are given by

$$
\begin{aligned}
v_{0}(s) & =\frac{1}{\mathcal{I}(s)} \int_{\Omega} \mathbf{E}_{0}(\mathbf{r}, s) \cdot \mathbf{J}(\mathbf{r}, s) d \mathbf{r} \\
v(s) & =\frac{1}{\mathcal{I}(s)} \int_{\Omega} \Delta \mathbf{E}(\mathbf{r}, s) \cdot \mathbf{J}(\mathbf{r}, s) d \mathbf{r}
\end{aligned}
$$

respectively, where $\Omega$ is the coil region. For an axially symmetric system and a coil with a uniform turns density, the coil current is given by (16) with (17), the free space field by (13), and the reflected electric field by (15). These equations, substituted into (24), give

$$
\begin{aligned}
v_{0}(s) & =\pi \mu_{0} s \mathcal{I}(s) \int_{0}^{\infty} \mathcal{F}(\kappa) d \kappa \\
v(s) & =\pi \mu_{0} s \mathcal{I}(s) \int_{0}^{\infty} \Gamma(\kappa, s)[\mathcal{J}(\kappa)]^{2} d \kappa
\end{aligned}
$$

where

$$
\begin{aligned}
\mathcal{F}(\kappa) & =n^{2} \int_{-b}^{b} \int_{-b}^{b} e^{-\kappa\left|z-z^{\prime}\right|} d z d z^{\prime}\left[a_{1}^{2} \chi\left(a_{1} \kappa\right)-a_{2}^{2} \chi\left(a_{2} \kappa\right)\right]^{2} \\
& =\frac{4 b n^{2}}{\kappa}\left[1-\frac{e^{-\kappa b} \sinh (\kappa b)}{b \kappa}\right]\left[a_{1}^{2} \chi\left(a_{1} \kappa\right)-a_{2}^{2} \chi\left(a_{2} \kappa\right)\right]^{2} .
\end{aligned}
$$

Hence, the time domain probe response is given by

$$
\begin{aligned}
V_{0}(t) & =L_{0} \frac{d I(t)}{d t} \\
& =\pi \mu_{0} \frac{d I(t)}{d t} \int_{0}^{\infty} \mathcal{F}(\kappa) d \kappa \\
V(t) & =\pi \mu_{0} \int_{0}^{\infty} \Phi^{\prime}(\kappa, t)[\mathcal{J}(\kappa)]^{2} d \kappa
\end{aligned}
$$

where $L_{0}=\pi \mu_{0} \int_{0}^{\infty} \mathcal{F}(\kappa) d \kappa$ is the free space self-inductance of the coil.

It is often useful to consider not just the induced EMF in the coil but also the integral of the EMF with respect to time. From (27), the integrated and negated signal due to induced current is

$$
\mathcal{V}(t)=-\pi \mu_{0} \int_{0}^{\infty} \Phi(\kappa, t)[\mathcal{J}(\kappa)]^{2} d \kappa
$$

Below, $\mathcal{V}(t)$ is calculated together with the variation of $V(t)$ for specific coil current transients.

In order to evaluate the induced EMF as a function of time, $\Phi^{\prime}(\kappa, t)$ has been found for two cases of interest. In the first case, the coil is assumed to be excited by a step current and in the second case, an exponentially rising current of the form given by (1). The first result is the limiting case of the second as the rise time goes to zero. 


\section{EVALUATION OF TRANSIENTS}

It is convenient to write the transverse electric reflection coefficient for a half-space of uniform conductivity $\sigma$, as

$$
\Gamma(\kappa, s)=\frac{2 \mu_{r}}{\mu_{r}+[\tau(\kappa) s+1]^{1 / 2}}-1
$$

where $\tau(\kappa)=\mu \sigma / \kappa^{2}$. With the reflection coefficient in this form the inverse Laplace transform may simply be looked up [13]. For brevity, the $\kappa$ dependence of $\tau$ is not shown explicitly in the development below but is assumed.

\section{A. Response Due to a Step Current}

Consider a coil carrying a current whose time variation is defined by $I(t)=I_{0} u(t)$ where $u(t)$ is a unit step function. Thus $u(t)=1$, for $t>0$ and $u(t)=0$ otherwise. Then the Laplace transform of the current is $\mathcal{I}(s)=I_{0} / s$ and

$$
\begin{aligned}
s \phi(\kappa, s) & =s \mathcal{I}(s) \Gamma(\kappa, s) \\
& =I_{0}\left[\frac{2 \mu_{r}}{\mu_{r}+(\tau s+1)^{1 / 2}}-1\right] .
\end{aligned}
$$

By rewriting this as

$$
s \phi(\kappa, s)=2 \mu_{r} I_{0} f(\tau s+1)-I_{0}
$$

and noting that the inverse transform of

$$
f(s)=\frac{1}{a+\sqrt{s}}
$$

is [13]

$$
F(t)=\frac{1}{\sqrt{\pi t}}-a e^{a^{2} t} \operatorname{erfc}(a \sqrt{t})
$$

where

$$
\operatorname{erfc}(z)=\frac{2}{\sqrt{\pi}} \int_{z}^{\infty} e^{-x^{2}} d x
$$

it is found that

$$
\begin{aligned}
\Phi^{\prime}(\kappa, t)= & \frac{2 \mu_{r} I_{0}}{\tau} e^{-t / \tau} \\
& \cdot\left[\sqrt{\frac{\tau}{\pi t}}-\mu_{r} e^{\mu_{r}^{2} t / \tau} \operatorname{erfc}\left(\mu_{r} \sqrt{\frac{t}{\tau}}\right)\right]-I_{0} \delta(t) .
\end{aligned}
$$

The integrated coil EMF and the magnetic field is expressed in terms of $\Phi(\kappa, t)$ in (28). One can determine $\Phi(\kappa, t)$ either by integrating (35) directly or by taking the inverse Laplace transform of the following [13]

$$
\frac{I_{0}}{s}\left[\frac{2 \mu_{r}}{\mu_{r}+[\tau s+1]^{1 / 2}}-1\right] .
$$

By either method, the result is $(t \geq 0)$

$$
\begin{aligned}
\Phi(\kappa, t)= & 2 I_{0} \frac{\mu_{r}}{\mu_{r}^{2}-1} \\
& \cdot\left[\mu_{r}-\operatorname{erf}\left(\sqrt{\frac{t}{\tau}}\right)-\mu_{r} e^{\left(\mu_{r}^{2}-1\right) t / \tau} \operatorname{erfc}\left(\mu_{r} \sqrt{\frac{t}{\tau}}\right)\right] \\
& -I_{0} u(t),
\end{aligned}
$$

where once again $\tau=\mu \sigma / \kappa^{2}$ and

$$
\operatorname{erf}(z)=\frac{2}{\sqrt{\pi}} \int_{0}^{z} e^{-x^{2}} d x .
$$

In the limit as the relative permeability approaches 1.0, (37) becomes

$$
\begin{aligned}
\Phi(\kappa, t)=2 I_{0} & {\left[\sqrt{\frac{t}{\pi \tau}} e^{-t / \tau}\right.} \\
+ & \left.\frac{1}{2}\left(1+\frac{2 t}{\tau}\right) \operatorname{erf}\left(\sqrt{\frac{t}{\tau}}\right)-\frac{t}{\tau}\right]-I_{0} u(t) .
\end{aligned}
$$

\section{B. Exponential Source Current}

With the coil current that varies exponentially as in (1), we have instead of (30)

$$
\begin{aligned}
s \phi_{0}(\kappa, s) & =s \mathcal{I}(s) \Gamma(\kappa, s) \\
& =I_{0} \frac{1}{\tau_{0} s+1}\left[\frac{2 \mu_{r}}{\mu_{r}+(\tau s+1)^{1 / 2}}-1\right] .
\end{aligned}
$$

A standard inverse Laplace transform [13] gives

$$
\begin{aligned}
\Phi_{0}^{\prime}(\kappa, t)= & \frac{2 I_{0} \mu_{r}}{\tau_{0}\left(\mu_{r}^{2}-\nu \tau\right)} \\
& \cdot\left\{e^{-t / \tau_{0}}\left[\mu_{r}-\sqrt{\nu \tau} \operatorname{erf}(\sqrt{\nu t})\right]\right. \\
& \left.\quad-\mu_{r} e^{\left(\mu_{r}^{2}-1\right) t / \tau} \operatorname{erfc}\left(\mu_{r} \sqrt{\frac{t}{\tau}}\right)\right\} \\
& -I_{0} \frac{1}{\tau_{0}} e^{-t / \tau_{0}}
\end{aligned}
$$

where

$$
\nu=\frac{1}{\tau}-\frac{1}{\tau_{0}} .
$$

The integrated response coil EMF and the magnetic field are found from

$$
\phi_{0}(\kappa, s)=I_{0}\left[\frac{1}{s}-\frac{\tau_{0}}{\tau_{0} s+1}\right]\left[\frac{2 \mu_{r}}{\mu_{r}+(\tau s+1)^{1 / 2}}-1\right]
$$

a form which allows us to express $\Phi_{0}$ in terms of functions defined earlier

$$
\Phi_{0}(\kappa, t)=\Phi(\kappa, t)-\tau_{0} \Phi_{0}^{\prime}(\kappa, t) .
$$

Clearly, if the system time constant $\tau_{0}$ is zero, (39) is recovered from (44).

\section{NORMALIZATION}

As with the coil impedance characteristic [4], it proves useful to introduce a normalization convention using the free-space coil self-inductance as a reference. With a step current excitation, the self-induced EMF in the coil in air is theoretically a delta function and has the form $I_{0} L_{0} \delta(t)$ for a transition at $t=0$. The normalized coil EMF and integrated response due to the reflected field are now defined as

$$
\begin{aligned}
& V_{n}(t)=V(t) / I_{0} L_{0} \quad \text { and } \\
& \mathcal{V}_{n}(t)=\mathcal{V}(t) / I_{0} L_{0} .
\end{aligned}
$$




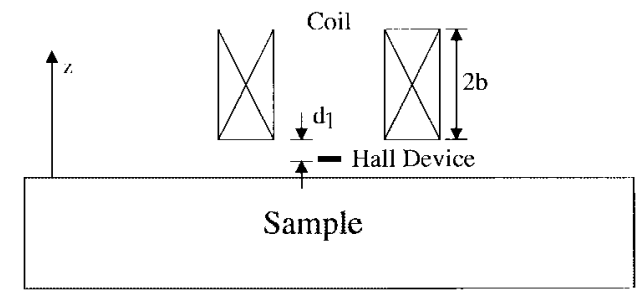

Fig. 5. Eddy-current probe cross section.

In the limit as $t \rightarrow 0(t>0)$ the integrated signal becomes

$$
\mathcal{V}_{n}(0)=\frac{\int_{0}^{\infty}[\mathcal{J}(\kappa)]^{2} d \kappa}{\int_{0}^{\infty} \mathcal{F}(\kappa) d \kappa}=k^{2}
$$

where $k$ is the probe coupling coefficient [14].

\section{EXPERIMENTAL PROCEDURE}

The normal component of the magnetic field on the axis of a coil in the presence of a thick conducting plate is to be measured. The coil is cylindrical and of rectangular cross section. Equation (1) governs the current flowing through the coil. The magnetic field is measured using a Hall device located on the coil axis, Fig. 5.

The coil current with its characteristic time constant $\tau_{0}$ is provided by a transconductance amplifier. The input to the transconductance amplifier is simply a square wave passed through a first-order low-pass filter as shown schematically in Fig. 1. The peak-to-peak coil current is about $32 \mathrm{~mA}$ while $\tau_{0}$ is equal to $275 \mu \mathrm{s}$. Measurements of the magnetic field are made by sampling the Hall device outputs at $20 \mu$ s intervals using a PC with an analog to digital converter card. Normally the PC will also be used to subtract a reference signal from all measurements. Such a reference could be the probe's transient response in air.

The Hall device sensitivity is temperature dependent but the asymptotic value of the incident field is fixed by the transconductance amplifier. The normal field at the Hall device may be calculated, (47), using the asymptotic coil current.

$$
\begin{aligned}
H_{z}= & K d_{2} \ln \left[\frac{a_{2}+\sqrt{a_{1}^{2}+d_{2}^{2}}}{a_{1}+\sqrt{a_{2}^{2}+d_{1}^{2}}}\right] \\
& -K d_{1} \ln \left[\frac{a_{2}+\sqrt{a_{1}^{2}+d_{2}^{2}}}{a_{1}+\sqrt{a_{2}^{2}+d_{1}^{2}}}\right]
\end{aligned}
$$

where

$$
K=\frac{n I_{0}}{2} \text {. }
$$

The distances between the Hall device and the bottom and top of the coil are $\mathrm{d}_{1}$ and $\mathrm{d}_{2}$, respectively. $I_{0}$ is the peak coil current. By using (47), the Hall device measurements are calibrated to yield normal magnetic field measurements.

Measurements have been made on aluminum and copper plates such that a half-space approximation is accurate, the aluminum plate had a thickness of $25 \mathrm{~mm}$, and the copper plate a thickness of $19 \mathrm{~mm}$. The samples have conductivities
TABLE I

Probe PARAMETERS

\begin{tabular}{ccc}
\hline Coil parameters & Coil 1 & Coil 2 \\
\hline Inner radius $(a 1)$ & $2.5 \mathrm{~mm}$ & $7.10 \mathrm{~mm}$ \\
Outer radius $(a 2)$ & $5.0 \mathrm{~mm}$ & $11.41 \mathrm{~mm}$ \\
Length $(2 b)$ & $2.5 \mathrm{~mm}$ & $5.0 \mathrm{~mm}$ \\
Number of turns & 100 & 2550 \\
\hline
\end{tabular}

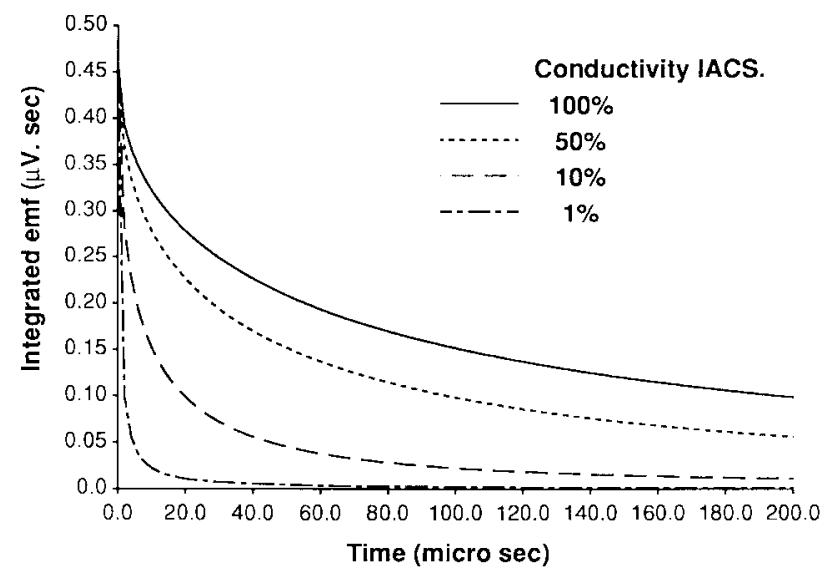

Fig. 6. Integrated eddy-current response for a range of conductivities with the source time constant $\tau_{0}=0$.

of $2.25 \times 10^{7} \mathrm{Sm}^{-1}$ and $5.80 \times 10^{7} \mathrm{Sm}^{-1}$, respectively. Coil 2, see Table I, was used with the Hall device $0.25 \mathrm{~mm}$ below the lower coil surface. A number of different probe lift-off values have been used by placing the probe on plastic shims of known thickness. The lift-off is the distance from the lower surface of the coil to the surface of the conductor.

\section{Numerical Results}

In Fig. 6, the normalized integrated coil EMF, $\mathcal{V}_{n}(t)$ is plotted as a function of time for various conductivities from $5.80 \times 10^{5} \mathrm{Sm}^{-1}$ to $5.80 \times 10^{7} \mathrm{Sm}^{-1}$. The coil parameters are given in Table I as coil 1 . The signal data have been calculated for the case of a step current, hence the time derivative represents the impulse response of the induced EMF due to the reflected field. Note that the initial signal at $t=0_{+}$, being equal to the square of the coupling parameter $k$, is independent of the conductivity of the workpiece and depends only on the coil parameters. The coupling coefficient decreases with liftoff. In the limit where the coil has zero axial length and zero lift-off, $k=1.0$. The coupling parameter represents a figure of merit for the coil-workpiece combination in the range zero to 1.0 .

The curves shown in Fig. 6 are geometrically similar, differing only in their time scale which increases in proportion to the conductivity of the workpiece. With the coil current given by (1), a current time constant $\tau_{0}=20 \mu \mathrm{s}$, the time dependence of $\mathcal{V}_{n}(t)$ for coil 1 is shown in Fig. 7. A finite time constant is necessary to avoid saturation of a current source, but it means that the coupling parameter cannot be read directly from the observed response. Fig. 8 shows the corresponding transients for a number of different source time constants from zero to $80 \mu \mathrm{s}$. 


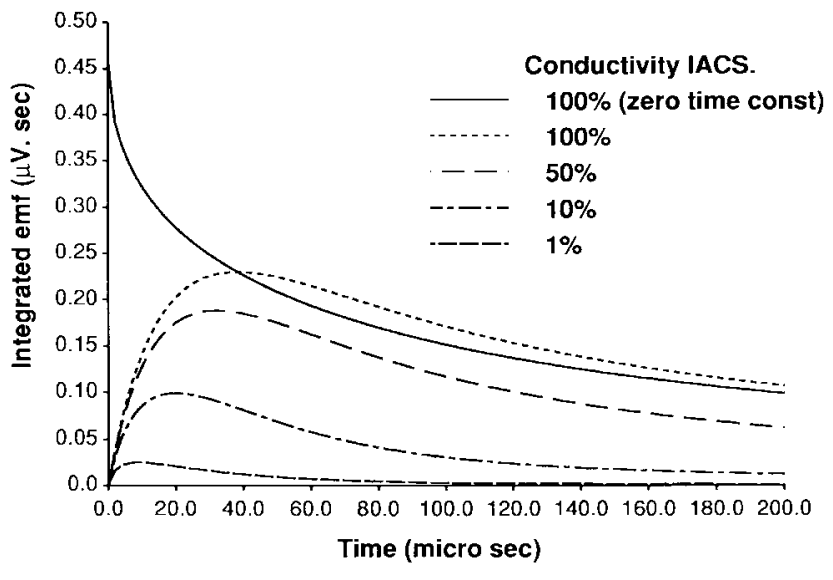

Fig. 7. Integrated eddy-current response for a range of conductivities with the source time constant $\tau_{0}=20 \mu \mathrm{s}$.

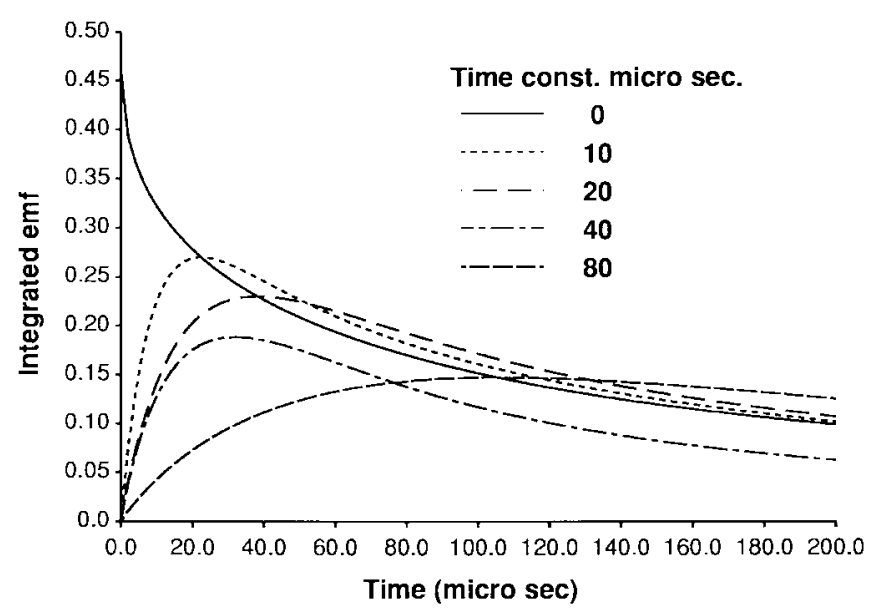

Fig. 8. Transients response for a number of different source time constants from zero to $80 \mu \mathrm{s}$.

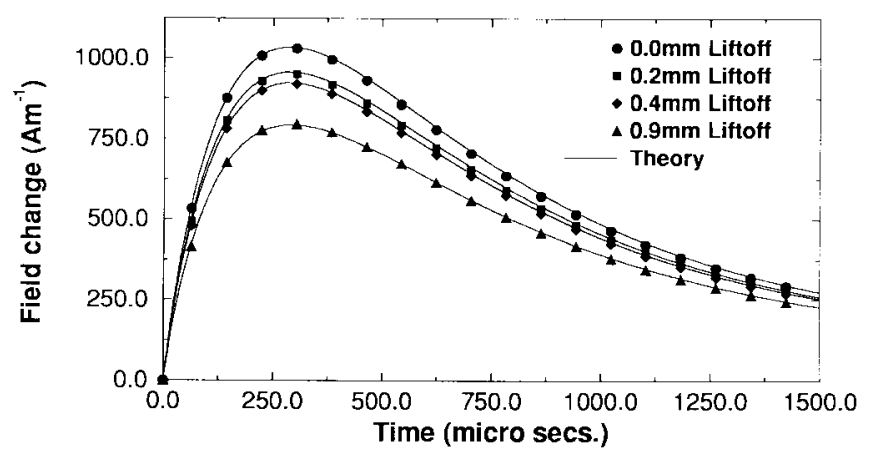

Fig. 9. Variation of Hall signal with time for various lift-off values. The sample was aluminum of conductivity $38.8 \%$ IACS. The source time constant was $275 \mu$ s.

Predictions of the normal field due to coil 2 (see Table I) have been made for the aluminum and copper plate samples over a range of lift-off values. The predictions for the aluminum, conductivity $2.25 \times 10^{7} \mathrm{Sm}^{-1}$, are compared with Hall measurements in Fig. 9. Similar comparisons have been made for the copper sample of conductivity $5.80 \times 10^{7} \mathrm{Sm}^{-1}$ in Fig. 10. Both sets of predictions show excellent agreement with experiment.

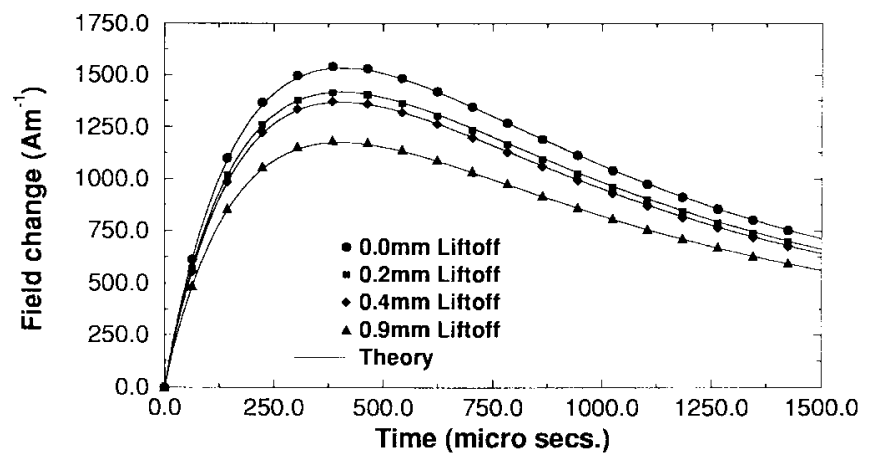

Fig. 10. Variation of Hall signal with time for various lift-off values. The sample was copper of conductivity $100 \%$ IACS. The source time constant was $275 \mu \mathrm{s}$.

\section{CONCLUSION}

The induced EMF of a coil due to transient eddy-currents in a half-space conductor have been calculated from closed-form integral expressions. The induced current decays with a characteristic time constant that is proportional to the conductivity of the material. The time constant is inversely proportional to the square of the spatial frequency $\kappa$ which means that the integrated decay time for a coil increases in proportion to the square of the coil dimension.

Predictions of induced EMF and the magnetic field have been calculated for a coil current that rises exponentially to a constant asymptotic value. Comparison of the time variation of the field with experimental measurements acquired using a Hall sensor show excellent agreement.

\section{REFERENCES}

[1] J. R. Bowler, "Transient eddy currents in layered media as a model of corrosion detection," Nondestructive Testing Evaluation, vol. 6, pp. 315-322, 1992.

[2] J. R. Wait, "Mutual coupling of loops lying on the ground," Geophys. Mag., vol. 13, pp. 290-296, 1954.

[3] P. Hammond, "The calculation of the magnetic field of rotating machines," Proc. Inst. Electr. Eng., Pt. 3(514S), pp. 508-515, 1962.

[4] C. V. Dodd and W. E. Deeds, "Analytical solutions to eddy-current probe-coil problems," J. Appl. Phys., vol. 39, no. 6, pp. 2829-2838, 1968.

[5] S. A. Teukolsky, W. H. Press, B. P. Flannery, and W. T. Vetterling, Numerical Recipes in C. Cambridge: Cambridge Univ. Press, 1988.

[6] J. R. Wait, "Propagation of electromagnetic pulses in a homogeneous conducting earth," Appl. Sci. Res., vol. 8, pp. 213-253, 1960.

[7] Y. M. Shkarlet and N. N. Lokshina, "Eddy current density during pulse excitation," Defektoskopiya, vol. 3, pp. 49-54, 1971.

[8] R. Ludwig and X. W. Dai, "Numerical and analytical modeling of pulsed eddy-currents in a conducting half-space," IEEE Trans. Magn., vol. 26, no. 1, pp. 299-307, 1990.

[9] J. R. Bowler, "Prediction and analysis of transient eddy-current probe signals," Rev. Progress Quantitative Nondestructive Evaluation, vol. 9A, pp. 287-293, 1990.

[10] J. Van Bladel, Electromagnetic Fields. New York: McGraw-Hill, 1964, p. 115.

[11] I. S. Gradshteyn and I. M. Ryzhik, Tables of Integrals, Series and Products. New York: Academic, 1980, sec. 6.612, p. 709.

[12] _ Tables of Integrals, Series and Products. New York: Academic, 1980 , sec. 6.561, p. 683.

[13] M. Abramowitz and I. Stegun, Handbook of Mathematical Functions with Formulas, Graphs, and Mathematical Tables. New York: Wiley, 1972, ch. 29.

[14] H. L. Libby, Introduction to Electromagnetic Nondestructive Test Methods. New York: Wiley, 1971, p. 157. 